\title{
Secrecy or transparency? The future of regulatory trial data
}

\author{
Beate Wieseler MSc PhD, Natalie McGauran
}

— Cite as: CMAJ 2017 February 6;189:E185-6. doi: 10.1503/cmaj.161088

See related article at www.cmaj.ca/lookup/doi/10.1503/cmaj.151104

$\mathbf{A}$ prerequisite for evidence-based medicine is the availability of complete and unbiased clinical trial information. Historically, journal publications have been the major route for disseminating the methods and results of clinical trials, and efforts to improve reporting have focused on improving journal publications, for example, through reporting guidelines. More recently, other routes, such as Web-based trial registries and documents prepared for regulatory agencies, are being considered for dissemination. A linked study by Maund and colleagues ${ }^{1}$ shows the value of a specific type of regulatory document, the clinical study report.

Clinical study reports are comprehensive documents prepared routinely for submission to regulatory authorities, which include detailed information on the design and results of clinical trials. Until recently, these reports were considered commercially confidential information and were kept concealed by pharmaceutical companies and regulatory authorities; these documents were thus not available to inform doctors and patients. However, in 2010 the European Medicines Agency (EMA) decided to make regulatory documents, including clinical study reports containing individual patient data, available upon request., ${ }^{2,3}$ Going even further, on Jan. 1, 2015, a policy on proactive publication of regulatory documents at the agency's website came into effect, which has resulted in these documents becoming publicly available for all drugs submitted for approval from that date onward. ${ }^{4}$ The first two clinical study reports were published by the EMA in October 2016. However, they do not include individual patient data because these data are to be published only in the second phase of the policy's implementation.

The authors of the linked study ${ }^{1}$ analyzed the benefits and harms of duloxetine for the treatment of stress urinary incontinence on the basis of four clinical trials, using clinical study reports (including individual patient data) requested from the EMA. In addition to end points related to the drug's benefits and general harms, they conducted an in-depth analysis of rare psychiatric adverse events that they considered particularly important, namely, suicidality and violence-related harms and their potential precursors (e.g., activation, emotional disturbance, psychotic events). These adverse events are not easily analyzed,

\section{KEY POINTS}

- Clinical study reports provide far more information relevant for trial evaluation than journal publications or trial registry reports but were previously kept concealed by pharmaceutical companies and regulatory authorities.

- In 2010, the European Medicines Agency made clinical study reports available upon request and, starting in October 2016, began routinely publishing clinical study reports of drugs submitted for approval.

- In terms of data transparency, Europe is ahead of the United States and Canada. The US Food and Drug Administration and Health Canada should join the European initiative to maximize the publicly available pool of clinical trial data.

because they might comprise multiple symptoms represented by various terms in adverse event coding systems. To estimate the rate of these harms, the adverse events reported had to be screened and terms combined. After considering these adverse events in their assessment of duloxetine, the authors concluded that the drug's harms outweigh its benefits in stress urinary incontinence.

In contrast, none of the journal publications or registry reports of the four trials under study provided specific information on psychiatric adverse events. This situation again shows the potential value of data held by regulatory agencies and confirms the findings of previous research: clinical study reports contain an abundance of information, including substantially more information relevant for trial evaluation than is available in journal publications and registry reports. ${ }^{5,6}$

Interestingly, the conclusion of the authors of the linked study ${ }^{1}$ contrasts with that of the EMA, even though the two assessments were based on the same evidence. The EMA concluded that duloxetine has a positive benefit-risk ratio for stress urinary incontinence, ${ }^{7}$ and the drug was approved in Europe in 2004. This discrepancy in conclusions seems to support the argument put forward by some data transparency opponents, who have stated that public access to clinical trial data would result in contradictions in data interpretation. But is this a problem? 
We don't think so - with all of the data being publicly available, different opinions can now be discussed openly.

Concerning the transparency of clinical trial information from regulatory authorities, Europe is ahead of the United States and Canada. In parallel with the EMA initiative, the update of the European Clinical Trial Regulation in 2014 was used to strengthen the legal basis for transparency. The law clarifies that clinical study reports in general should not be considered commercially confidential information and must be made publicly available after a drug's approval. ${ }^{8}$

Although the US Food and Drug Administration and Health Canada may release clinical trial information in individual cases, so far they do not consider making these data publicly available on a routine basis. ${ }^{9}$ However, the US Institute of Medicine's proposals for the responsible sharing of clinical trial data refer in particular to clinical study reports and individual patient data, and this agency has called upon regulatory authorities to actively support this initiative. ${ }^{10}$

The treasure of data available from the EMA should be used to improve patient care. However, according to information provided by the EMA about requests for data under its new policy, the pharmaceutical industry has so far submitted the largest number of requests for data, ${ }^{11}$ which indicates that clinical study reports are currently used predominantly for commercial purposes. Moreover, although industry researchers are familiar with this type of data, many nonindustry researchers have little experience in analyzing them and may not be fully aware of the potential they offer.

The authors of the linked study were able to analyze harms consisting of multiple symptoms only because they had access to individual patient data; this again shows that full clinical study reports including individual patient data are indispensable to answer relevant research questions. Timely implementation of the second phase of the EMA initiative (public access to individual patient data) is thus desirable.

What should the next steps be? First, data that are made available must be used. For instance, analyzing clinical data published on the EMA's website should become a standard approach for systematic reviewers. At the same time, feasible models for the use of individual patient data are needed, models that protect patient privacy while preserving the information density of the data to make full use of clinical trial results. Moreover, the US Food and Drug Administration, Health Canada and other regulatory agencies should join the Institute of Medicine and EMA initiatives to maximize the publicly available pool of clinical trial data. Last but not least, the pharmaceutical industry should refrain from obstructing transparency gains, for example, through the new European Union trade secrets directive. ${ }^{12}$
The recent steps toward increased transparency of clinical trial information can be considered a true innovation in health care. Used appropriately, drug development and drug assessment based on publicly available, comprehensive clinical trial data have the most potential to improve patient care.

\section{References}

1. Maund E, Schow Guski L, Gøtzsche PC. Considering benefits and harms of duloxetine for treatment of stress urinary incontinence: a meta-analysis of clinical study reports. CMAJ 2017;189:E194-203.

2. European Medicines Agency policy on access to documents (related to medicinal products for human and veterinary use) - policy/0043. EMA/110196/2006. London (UK): European Medicines Agency; 2010. Available: www.ema.europa.eu/ docs/en_GB/document_library/Other/2010/11/WC500099473.pdf (accessed 2016 Aug. 19).

3. Gøtzsche PC, Jørgensen AW. Opening up data at the European Medicines Agency. BMJ 2011;342:d2686.

4. European Medicines Agency policy on publication of clinical data for medicinal products for human use - policy/0070. EMA/240810/2013. London (UK): European Medicines Agency; 2014. Available: www.ema.europa.eu/docs/en_GB/ document_library/Other/2014/10/WC500174796.pdf (accessed 2016 Aug. 19).

5. Wieseler B, Kerekes MF, Vervoelgyi V, et al. Impact of document type on reporting quality of clinical drug trials: a comparison of registry reports, clinical study reports, and journal publications. BMJ 2012;344:d8141.

6. Doshi $\mathrm{P}$, Jefferson $\mathrm{T}$, Del Mar C. The imperative to share clinical study reports: recommendations from the Tamiflu experience. PLoS Med 2012;9:e1001201.

7. Yentreve: EPAR [European public assessment report] - scientific discussion. London (UK): European Medicines Agency; 2005. Available: www.ema.europa.eu/ docs/en_GB/document_library/EPAR_-_Scientific_Discussion/human/000545/ WC500045641.pdf (accessed 2016 Aug. 19).

8. Mayor S. New European legislation on clinical trials is published. BMJ 2014;348:g4102.

9. Persaud N, Doshi P. North American regulatory agencies can and should make clinical trial data publicly available. CMAJ 2016;188:96-7.

10. Institute of Medicine. Sharing clinical trial data: maximizing benefits, minimizing risk. Washington (DC): National Academies Press; 2015.

11. Doshi P, Jefferson T. The first 2 years of the European Medicines Agency's policy on access to documents: secret no longer. JAMA Intern Med 2013;173:380-2.

12. Trade secrets directive will threaten access to medicines safety and efficacy data [media statement]. Amsterdam (The Netherlands): Health Action International; 2016; Available: http://haiweb.org/publication/trade-secrets-directive-will -threaten-access-medicines-safety-efficacy-data/ (accessed 2016 Aug. 22).

Competing interests: The production of unbiased health technology assessment reports requires access to all of the relevant data on the particular topic under investigation. Therefore, the authors support public access to clinical study reports. No other competing interests were declared.

This article was solicited and has been peer reviewed.

Affiliation: Institute for Quality and Efficiency in Health Care, Cologne, Germany

Contributors: Beate Wieseler drafted the article and Natalie McGauran revised it critically for important intellectual content. Both of the authors gave final approval of the version to be published and agreed to act as guarantors of the work.

Correspondence to: Beate Wieseler, beate.wieseler@iqwig.de 